International Journal of Bifurcation and Chaos, Vol. 12, No. 12 (2002) 2897-2905

(c) World Scientific Publishing Company

\title{
AN EXPERIMENTAL SUPPRESSION OF SPATIAL INSTABILITY IN ONE-WAY OPEN COUPLED CHUA'S CIRCUITS
}

\author{
TAKUYA IMAI, KEIJI KONISHI*, \\ HIDEKI KOKAME and KENTARO HIRATA \\ Department of Electrical and Electronic Systems, \\ Osaka Prefecture University, \\ 1-1 Gakuen-cho, Sakai, Osaka 599-8531 Japan \\ *kkonishi@fun.ac.jp
}

Received February 4, 2002; Revised February 13, 2002

\begin{abstract}
This letter shows an experimental suppression of spatial instability in one-way open coupled Chua's circuits. A continuous-time version of the decentralized delayed feedback method is used for a suppression scheme. Furthermore, we confirm that the control method also succeeds in suppressing chaotic behavior in the coupled circuits.
\end{abstract}

Keywords: Chua's circuit; delayed-feedback control; spatial instability.

\section{Introduction}

Controlling chaos has attracted a growing interest in the field of nonlinear science [Fradkov \& Pogromsky, 1998; Chen \& Dong, 1998]. Pyragas proposed a delayed feedback control (DFC) method [Pyragas, 1992], which does not require a reference signal corresponding to the desired unstable periodic orbits. The DFC method has been widely applied to real systems, since it is a practical scheme for experimental situations [Chen \& Dong, 1998]. In recent years, an increasing interest has been devoted to the study of spatiotemporal chaos and its control [Astakhov et al., 1995; Hu et al., 1997; Konishi et al., 1998, 2000a].

There has been some interest in a wide variety of complex spatiotemporal behavior of coupled map lattices (CMLs) [Kaneko, 1993]. The one-way open CML [Kaneko, 1985] is well known as a typical open flow model. In this CML the spatial instability was discovered by Kaneko [1985], and it was examined in detail theoretically [Yamaguchi, 1997] and numerically [Willeboordse \& Kaneko, 1995]. Recently, the mechanism of the instability in this CML was clarified by the $H_{\infty}$-norm concept, which plays important roles in the robust control theory [Konishi et al., 1999]. Konishi, Kokame and Hirata reported that the decentralized delayed feedback control (DDFC) method can stabilize chaotic behavior in a one-way open CML [Konishi et al., 1998, 2000a]. Imai et al. observed the instability in the electronic CML circuits, and demonstrated the suppression of the instability by the DDFC method [Imai et al., 2002]. Konishi et al. [2000b] verified that the mechanism of the spatial instability in the CML can be extended to continuoustime systems. The instability was observed in coupled diode resonator circuits [Johnson et al., 1995] and coupled Chua's circuits [Konishi et al., 2001]. Furthermore, it was reported that the LMI-based $H_{\infty}$ control can suppress the instability in the oneway coupled Lorenz systems [Konishi et al., 2002].

*Address for correspondence: Department of Complex Systems, Future University-Hakodate, Hakodate, Hokkaide 041-8655, Japan. 
The present letter provides an experimental evidence for suppression of the spatial instability in a continuous-time system (i.e. the one-way open coupled Chua's circuit [Kapitaniak et al., 1994; Konishi et al., 2001]). We adopt a continuous-time version of the DDFC method for suppression of the instability. In addition, it is shown that this method succeeds in suppressing chaotic behavior in the coupled circuits.

\section{Spatial Instability}

Let us consider one-way open coupled Chua's circuits illustrated in Fig. 1 [Kapitaniak et al., 1994]. The state equations for the circuits are as follows

$$
\left\{\begin{array}{l}
C_{1} \frac{d x_{1}^{(i)}(t)}{d t}=\frac{1}{R}\left\{x_{2}^{(i)}(t)-x_{1}^{(i)}(t)\right\}+g\left(x_{1}^{(i)}(t)\right) \\
C_{2} \frac{d x_{2}^{(i)}(t)}{d t}=\frac{1}{R}\left\{x_{1}^{(i)}(t)-x_{2}^{(i)}(t)\right\}+x_{3}^{(i)}(t)+\frac{1}{r}\left\{x_{2}^{(i-1)}(t)-x_{2}^{(i)}(t)\right\} \\
L \frac{d x_{3}^{(i)}(t)}{d t}=-x_{2}^{(i)}(t)
\end{array}\right.
$$

for $i=1,2, \ldots, N$. Each circuit consists of the nonlinear resistor $N_{R}$, the linear resistor $R$, the inductor $L$, and the two capacitors $C_{1}$ and $C_{2}$. The resistor $R$ is used as a system parameter. The voltages across $C_{1}, C_{2}$, and the current through $L$ of the $i$ th Chua's circuit are denoted by the state variables $x_{1}^{(i)}(t), x_{2}^{(i)}(t)$, and $x_{3}^{(i)}(t)$, respectively. The nonlinear resistor $N_{R}$ is defined by

$$
g\left(x_{1}\right)=m_{0} x_{1}+\frac{1}{2}\left(m_{1}-m_{0}\right)\left\{\left|x_{1}+B_{p}\right|-\left|x_{1}-B_{p}\right|\right\} .
$$

This nonlinear resistor has three linear segments: the slopes in the inner and outer segments are $m_{1}$ and $m_{0}$, respectively. The voltages $\pm B_{p}[\mathrm{~V}]$ denote the breakpoints of the segments. Each circuit is coupled to the upper circuit by the voltage buffer and the coupling resistor $r$, which realize a one-way connection. The upper edge is set to $x_{2}^{(0)} \equiv 0[\mathrm{~V}]$. The system size $N$ is fixed at $N=16$ throughout this letter.

If $m_{1}<-(1 / R)<m_{0}$ is satisfied, there coexist the three fixed points in each circuit. In this letter, for the purpose of simplicity, we focus on the homogeneous solution,

$$
\begin{aligned}
{\left[\begin{array}{llllll}
\mathbf{x}(1) & \mathbf{x}(2) & \cdots & \mathbf{x}(i) & \cdots & \mathbf{x}(N)
\end{array}\right] } \\
=\left[\begin{array}{llllll}
\mathbf{P} & \mathbf{P} & \cdots & \mathbf{P} & \cdots & \mathbf{P}
\end{array}\right]
\end{aligned}
$$



Fig. 1. One-way open coupled Chua's circuits with controllers. The symbol C represents the delayed feedback controller. 
where $\mathbf{x}(i)=\left[\begin{array}{lll}x_{1}^{(i)}(t) & x_{2}^{(i)}(t) & x_{3}^{(i)}(t)\end{array}\right]^{T}$ and $\mathbf{P}=$ $\left[\begin{array}{lll}x_{f} & 0 & -x_{f} / R\end{array}\right]^{T}$. The symbol $x_{f}$ is $R B_{p}\left(m_{0}-\right.$ $\left.m_{1}\right) /\left(m_{0} R+1\right)$. The linearized dynamics of coupled circuit (1) around solution (2) is described by

$$
\left\{\begin{array}{l}
\frac{d \mathbf{z}^{(i)}(t)}{d t}=\mathbf{A} \mathbf{z}^{(i)}(t)+\mathbf{b} z_{2}^{(i-1)}(t), \\
z_{2}^{(i)}(t)=\mathbf{c} \mathbf{z}^{(i)}(t)
\end{array}\right.
$$

where

$$
\begin{gathered}
\mathbf{z}^{(i)}(t)=\left[\begin{array}{ccc}
z_{1}^{(i)}(t) & z_{2}^{(i)}(t) & z_{3}^{(i)}(t)
\end{array}\right]^{T}:=\mathbf{x}^{(i)}(t)-\mathbf{P}, \\
\mathbf{A}:=\left[\begin{array}{ccc}
-\frac{1}{C_{1}}\left(\frac{1}{R}+m_{0}\right) & \frac{1}{C_{1} R} & 0 \\
\frac{1}{C_{2} R} & -\frac{1}{C_{2}}\left(\frac{1}{R}+\frac{1}{r}\right) & \frac{1}{C_{2}} \\
0 & -\frac{1}{L} & 0
\end{array}\right], \\
\mathbf{b}:=\left[\begin{array}{lll}
0 & \frac{1}{r C_{2}} & 0
\end{array}\right]^{T}, \quad \mathbf{c}:=\left[\begin{array}{lll}
0 & 1 & 0
\end{array}\right] .
\end{gathered}
$$

The state $\mathbf{z}^{(i)}(t)$ is a deviation from the fixed point $\mathbf{P}$ for the circuit $i$. We remark that system (3) has input $z_{2}^{(i-1)}(t)$ and output $z_{2}^{(i)}(t)$ signals. Let us focus on system (3) in the frequency domain:

$$
\left\{\begin{array}{l}
s \mathbf{Z}^{(i)}(s)=\mathbf{A} \mathbf{Z}^{(i)}(s)+\mathbf{b} Z_{2}^{(i-1)}(s) \\
Z_{2}^{(i)}(s)=\mathbf{c} \mathbf{Z}^{(i)}(s)
\end{array}\right.
$$

where

$$
\begin{gathered}
s \mathbf{Z}^{(i)}(s):=\mathcal{L}\left[d \mathbf{z}^{(i)}(t) / d t\right], \quad \mathbf{Z}^{(i)}(s):=\mathcal{L}\left[\mathbf{z}^{(i)}(t)\right] \\
Z_{2}^{(i)}(s):=\mathcal{L}\left[z_{2}^{(i)}(t)\right], \quad Z_{2}^{(i-1)}(s):=\mathcal{L}\left[z_{2}^{(i-1)}(t)\right] .
\end{gathered}
$$

The symbol $\mathcal{L}$ denotes the Laplace transform function. The input-output relation of system (4) is given by $Z_{2}^{(i)}(s)=G(s) Z_{2}^{(i-1)}(s)$. The transfer function, described by

$$
\begin{aligned}
G(s) & =\mathbf{c}\left(s \mathbf{I}_{3}-\mathbf{A}\right)^{-1} \mathbf{b} \\
& =k \frac{s^{2}+\beta_{1} s}{s^{3}+\alpha_{2} s^{2}+\alpha_{1} s+\alpha_{0}}
\end{aligned}
$$

represents the input-output relation, where

$$
\begin{aligned}
k & :=\frac{1}{r C_{2}}, \quad \beta_{1}:=\frac{1+m_{0} R}{R C_{1}} \\
\alpha_{0} & :=\frac{1}{L C_{1} C_{2}}\left(m_{0}+\frac{1}{R}\right) \\
\alpha_{1} & :=\frac{1}{C_{2}}\left[\frac{m_{0}}{C_{1}}\left(\frac{1}{R}+\frac{1}{r}\right)+\frac{1}{R r C_{1}}+\frac{1}{L}\right], \\
\alpha_{2} & :=\frac{1}{C_{1}}\left(m_{0}+\frac{1}{r}\right)+\frac{1}{C_{2}}\left(\frac{1}{R}+\frac{1}{r}\right) .
\end{aligned}
$$

Now, we introduce the spatiotemporal stability based on the $H_{\infty}$-norm of $G(s)$ [Konishi et al., 2000b].

Definition 1 [Konishi et al., 2000b]. The spatiotemporal stability of homogeneous solution (2) of system (1) is classified into three states.

(i) If $G(s)$ is unstable, it is Temporally Unstable $(\mathrm{TU})$.

(ii) If $G(s)$ is stable and the $H_{\infty}$-norm of $G(s)$ is less than 1, it is Temporally-Spatially Stable (TSS).

(iii) If $G(s)$ is stable and the $H_{\infty}$-norm of $G(s)$ is greater than 1 , it is Temporally Stable and Spatially Unstable (TSSU).

The definition of the $H_{\infty}$-norm is shown in the Appendix. The spatial instability occurs only when the homogeneous solution is TSSU. Its mechanism is due to the fact that a tiny external noise at the upper circuits grows downstream in open flow systems [Konishi et al., 1998, 2000b, 2001].

\section{Suppression of Spatial Instability}

The spatial instability and chaotic behavior in real open flow systems are undesirable phenomena from an engineering viewpoint; therefore, it would be necessary to eliminate them. In this section we propose a method to suppress the spatial instability. From Definition 1, we notice that the spatial instability never occurs when the homogeneous solution is TSS (i.e. $G(s)$ is stable and the $H_{\infty}$-norm of $G(s)$ is less than 1). In order to suppress the spatial instability, we add the DFC-based controller to every circuit. The control signal $u^{(i)}(t)$ is added to the capacitor $C_{2}$ at the circuit $i$ as a feedback current (see Fig. 1). Thus, we have the state equations of the controlled circuit, 


$$
\left\{\begin{array}{l}
C_{1} \frac{d x_{1}^{(i)}(t)}{d t}=\frac{1}{R}\left\{x_{2}^{(i)}(t)-x_{1}^{(i)}(t)\right\}+g\left(x_{1}^{(i)}(t)\right) \\
C_{2} \frac{d x_{2}^{(i)}(t)}{d t}=\frac{1}{R}\left\{x_{1}^{(i)}(t)-x_{2}^{(i)}(t)\right\}+x_{3}^{(i)}(t)+\frac{1}{r}\left\{x_{2}^{(i-1)}(t)-x_{2}^{(i)}(t)\right\}+u^{(i)}(t), \\
L \frac{d x_{3}^{(i)}(t)}{d t}=-x_{2}^{(i)}(t) .
\end{array}\right.
$$

The control signal $u^{(i)}(t)$ is proportional to the difference between the past $x_{2}^{(i)}(t-T)$ and current $x_{2}^{(i)}(t)$ voltages:

$$
u^{(i)}(t)=\frac{1}{R_{u}}\left\{x_{2}^{(i)}(t-T)-x_{2}^{(i)}(t)\right\} .
$$

The gain of $u^{(i)}(t)$ can be varied by the resistance $R_{u}$, and $T$ is the delay time. The procedure mentioned in the previous section allows us to obtain the input-output relation of controlled circuit (6), $Z_{2}^{(i)}(s)=\bar{G}(s) Z_{2}^{(i-1)}(s)$. The transfer function is described by

$$
\bar{G}(s)=k \frac{s^{2}+\beta_{1} s}{s^{3}+\bar{\alpha}_{2} s^{2}+\bar{\alpha}_{1} s+\alpha_{0}},
$$

where

$$
\begin{aligned}
& \bar{\alpha}_{1}=\alpha_{1}+\frac{m_{0} R+1}{C_{1} C_{2} R R_{u}}\left(1-e^{-s T}\right), \\
& \bar{\alpha}_{2}=\alpha_{2}+\frac{1}{C_{2} R_{u}}\left(1-e^{-s T}\right) .
\end{aligned}
$$

If the gain resistance $R_{u}$ and the delay time $T$ satisfy

(1) $\bar{G}(s)$ is stable,

(2) $H_{\infty}$-norm of $\bar{G}(s)$ is less than 1 ,

then we can suppress the spatial instability. The design of the gain and the delay time can be considered as a DFC-based $H_{\infty}$ control problem. Unfortunately, the robust control theory cannot give us an answer to this problem at the present stage. In other words, there is no systematic procedure for design of the feedback gain and the delay time. There is room for further investigation in the field of control theory.

\section{Experimental Results}

This section explains how to realize the one-way open coupled Chua's circuits and the controllers by electronic devices. We then show an experimental suppression of the spatial instability and chaotic behavior in the coupled circuits. Throughout this letter the circuit parameters are fixed as follows: $C_{1}=100[\mathrm{nF}], C_{2}=1000[\mathrm{nF}], L=200[\mathrm{mH}]$, $m_{0}=-0.40[\mathrm{mS}], m_{1}=-0.77[\mathrm{mS}], B_{p}=1.0[\mathrm{~V}]$.

\subsection{Implementation}

We implement the circuits and the controllers by electronic devices as illustrated in Fig. 1. The opamps TL084 are used for the voltage buffer and the nonlinear resistor $N_{R}$. This resistor $N_{R}$ can be realized by two op-amps and six linear resistors [Kennedy, 1994]. The real inductors inevitably have an inner resistance; therefore, in order to realize the ideal inductor, we employ the impedance converter which is given by $\dot{Z}_{L}=j \omega\left(C_{4} R_{1} R_{3} R_{5} / R_{2}\right)$ (see Fig. 2). The parameters are set to $R_{1}=R_{2}=$ $R_{3}=1.0[\mathrm{k} \Omega], C_{4}=100[\mathrm{nF}]$, and $R_{5}=2.0[\mathrm{k} \Omega]$, then we obtain the impedance $\dot{Z}_{L}=j \omega 200 \times 10^{-3}$, which is equivalent to the ideal inductor $L=200$ $[\mathrm{mH}]$. Figure 3 illustrates the delayed feedback controller. The circuit state $x_{2}^{(i)}(t)$ is input to the $i$ th controller as a voltage signal. A bucket brigade delay line (National Panasonic, type MN3011) with 3328 stages allows us to realize a delayed feedback signal $x_{2}^{(i)}(t-T)$. The delay time $T$ can be varied



Fig. 2. Impedance converter. 


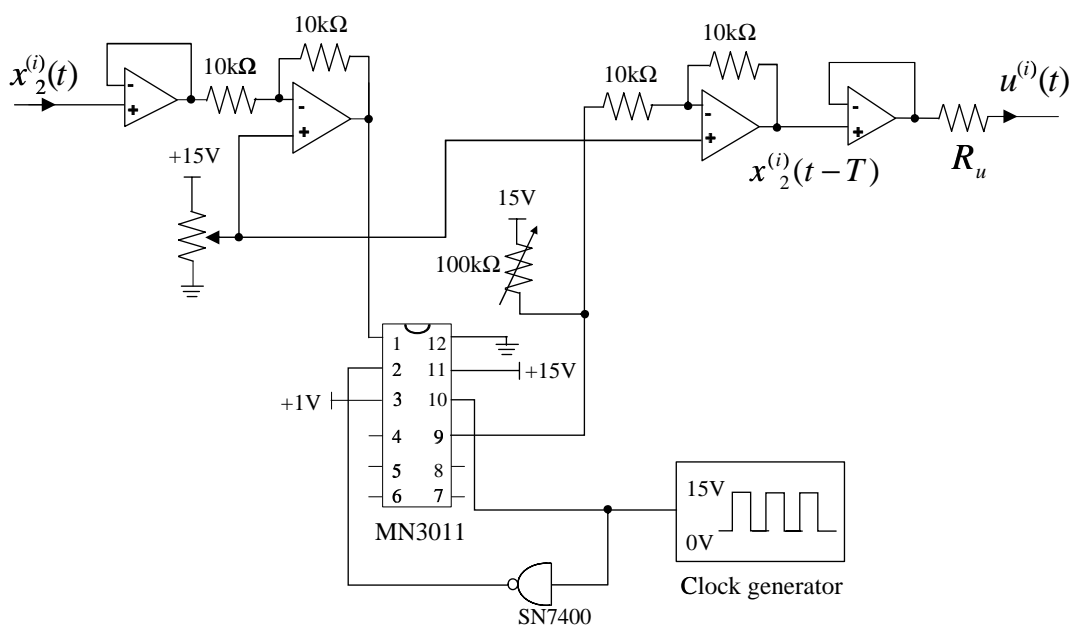

Fig. 3. Implementation of the $i$ th controller.

in the range from 1.98 to 166.40 [msec] by changing the frequency of an external clock generator. The feedback gain in Eq. (7) can be set by the resistor $R_{u}$. The control signal $u^{(i)}(t)$ is output from the $i$ th controller as a current signal.

\subsection{Suppression of spatial instability and chaos}

We consider coupled system (1) without control for a parameter set $(R, r)=(1.92[\mathrm{k} \Omega], 5.15[\mathrm{k} \Omega])$, and check the spatiotemporal stability by a systematic procedure previously proposed in [Konishi et al., 2001]. The gain $|G(j \omega)|$ is plotted against the frequency $f=\omega / 2 \pi$ in Fig. $4(\mathrm{a})$; it is confirmed that the peak gain, that is the $H_{\infty}$-norm of $G(s)$, is greater than 1 . This result shows that homogeneous solution (2) of coupled system (1) is TSSU for this parameter set. In Fig. 4(b) it is shown that the amplitude of oscillation increases with an increase in the number of circuits. This amplitude increase is the spatial instability phenomenon.

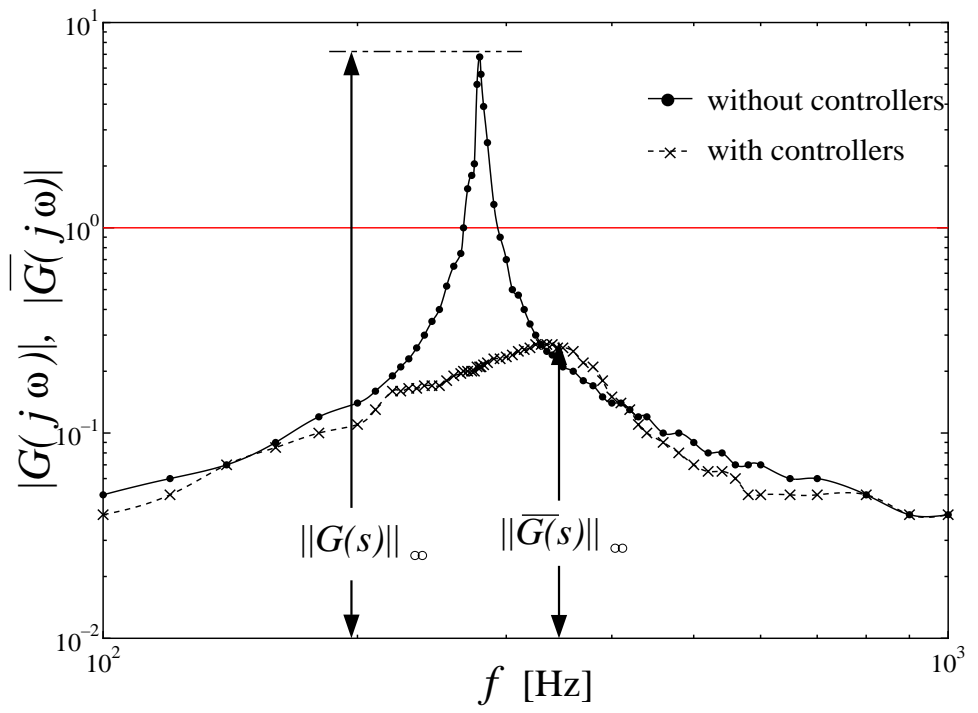

(a)

Fig. 4. Spatial instability in TSSU regime and its suppression $(R=1.92[\mathrm{k} \Omega], r=5.15[\mathrm{k} \Omega])$. (a) Gain diagram. (b) Spatial instability. (c) Suppression of the spatial instability $\left(R_{u}=2.0[\mathrm{k} \Omega], T=3.96[\mathrm{msec}]\right)$. 


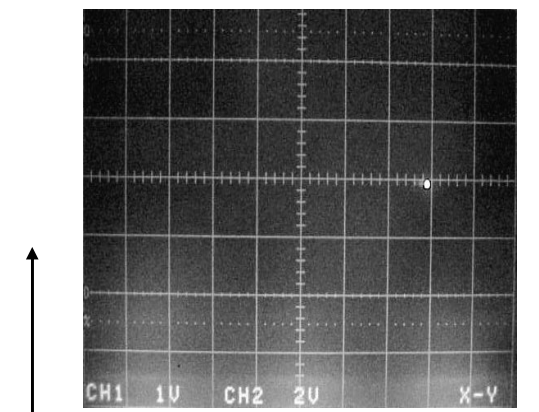

circuit 1



circuit 10

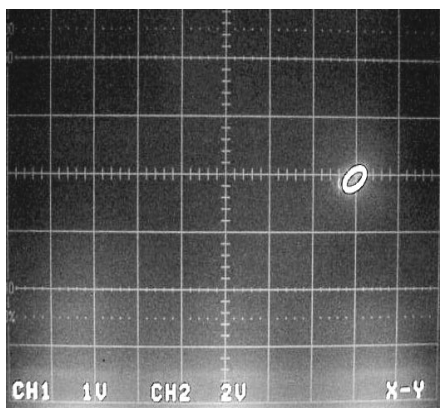

circuit 6



circuit 13

$x_{1}^{(i)}(t)$



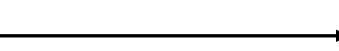

(b)

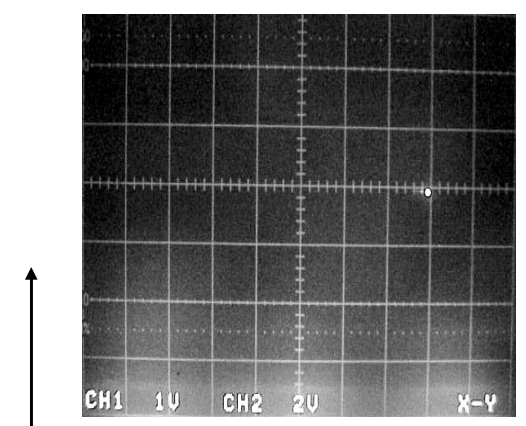

circuit 1

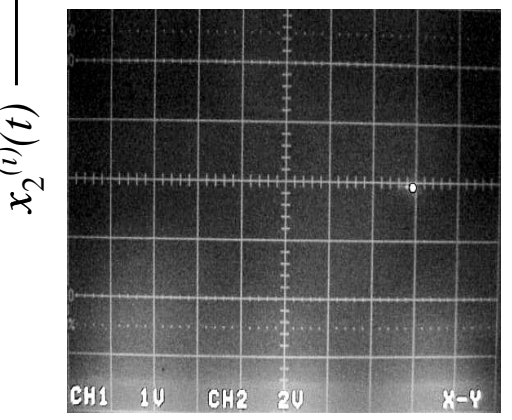

circuit 10



circuit 6



circuit 13

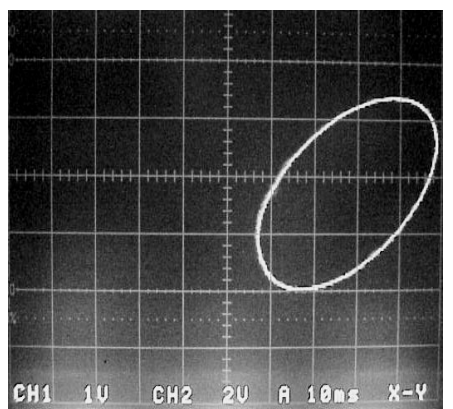

circuit 8

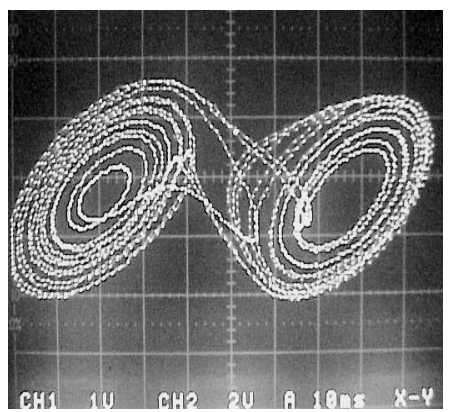

circuit 16

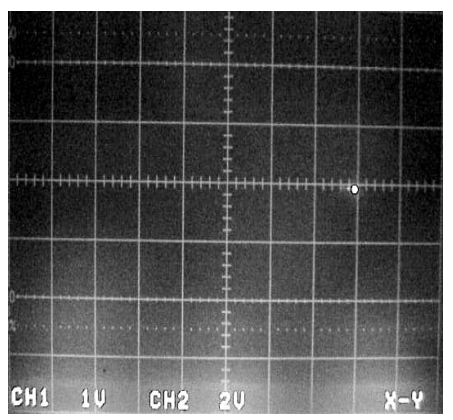

circuit 8

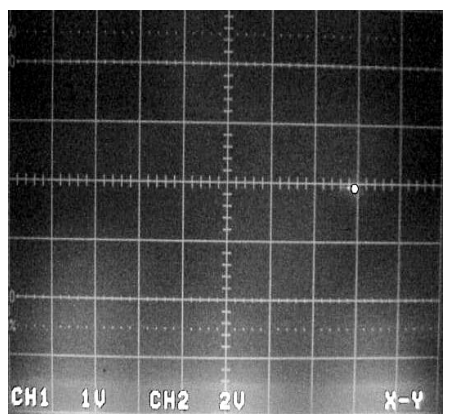

circuit 16

(c)

Fig. 4. (Continued) 


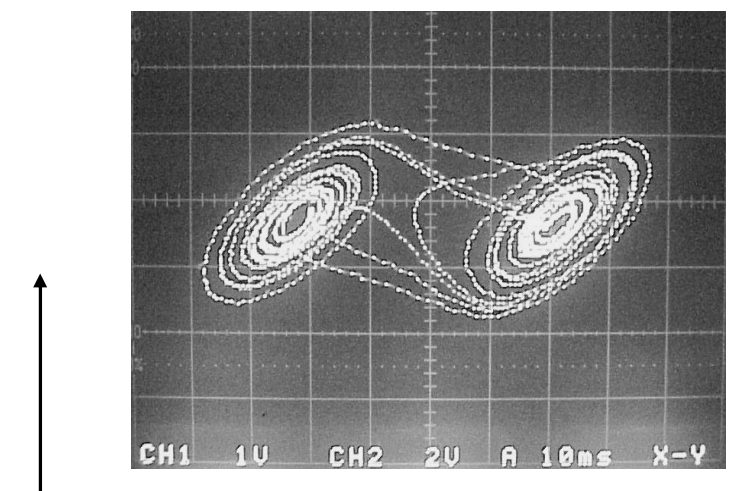

circuit 1

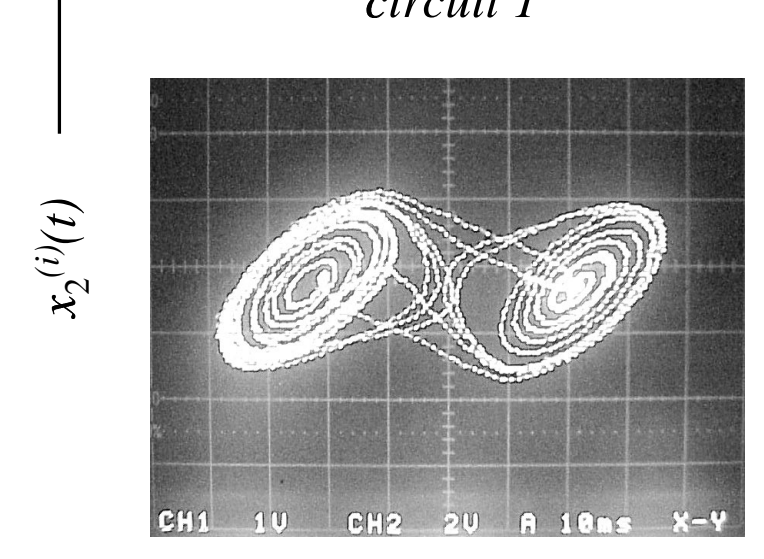

circuit 9

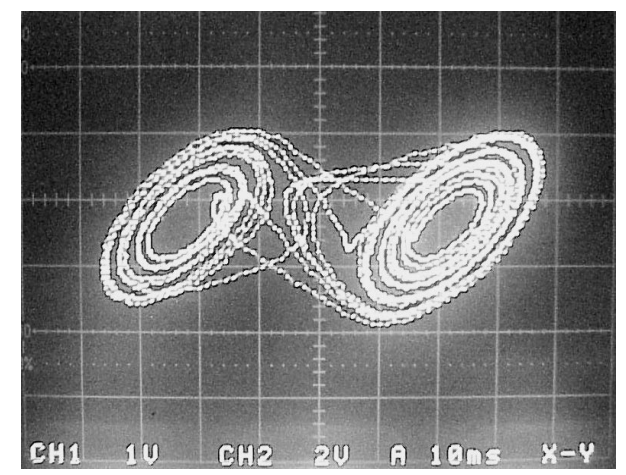

circuit 5

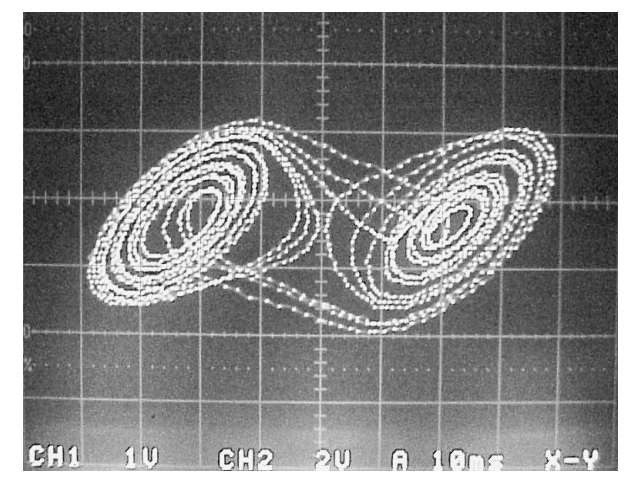

circuit 16

(a)

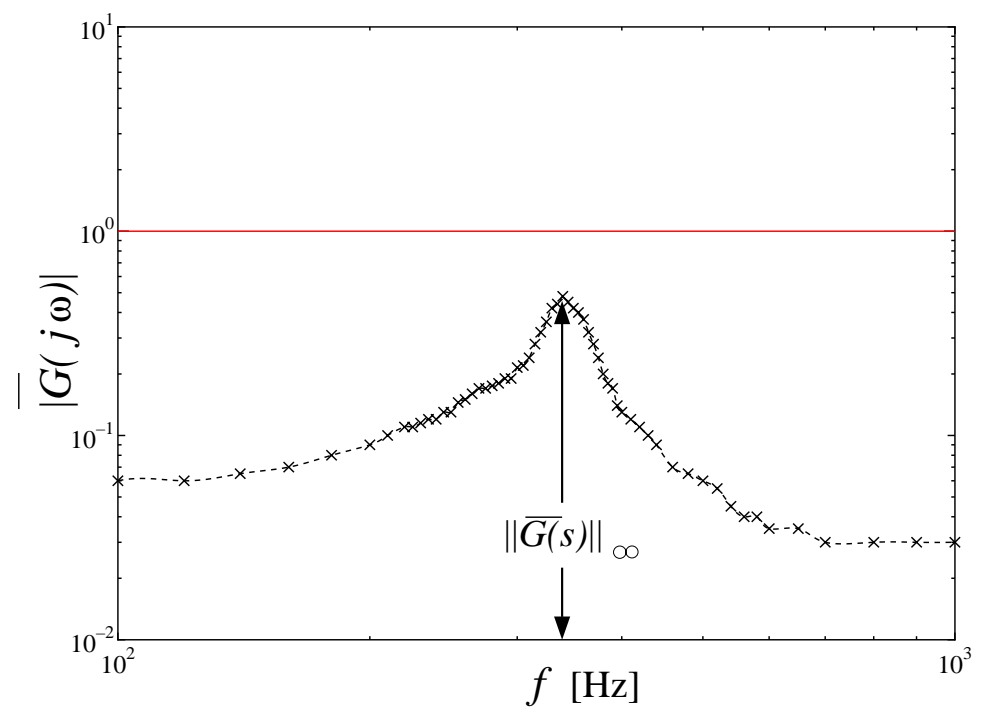

(b)

Fig. 5. Chaotic behavior in TU regime and its suppression $(R=1.8[\mathrm{k} \Omega], r=8.0[\mathrm{k} \Omega])$. (a) Chaotic behavior in TU regime. (b) Gain diagram. (c) Suppression of the chaotic behavior in TU regime $\left(R_{u}=2.2[\mathrm{k} \Omega], T=3.5[\mathrm{msec}]\right)$. 

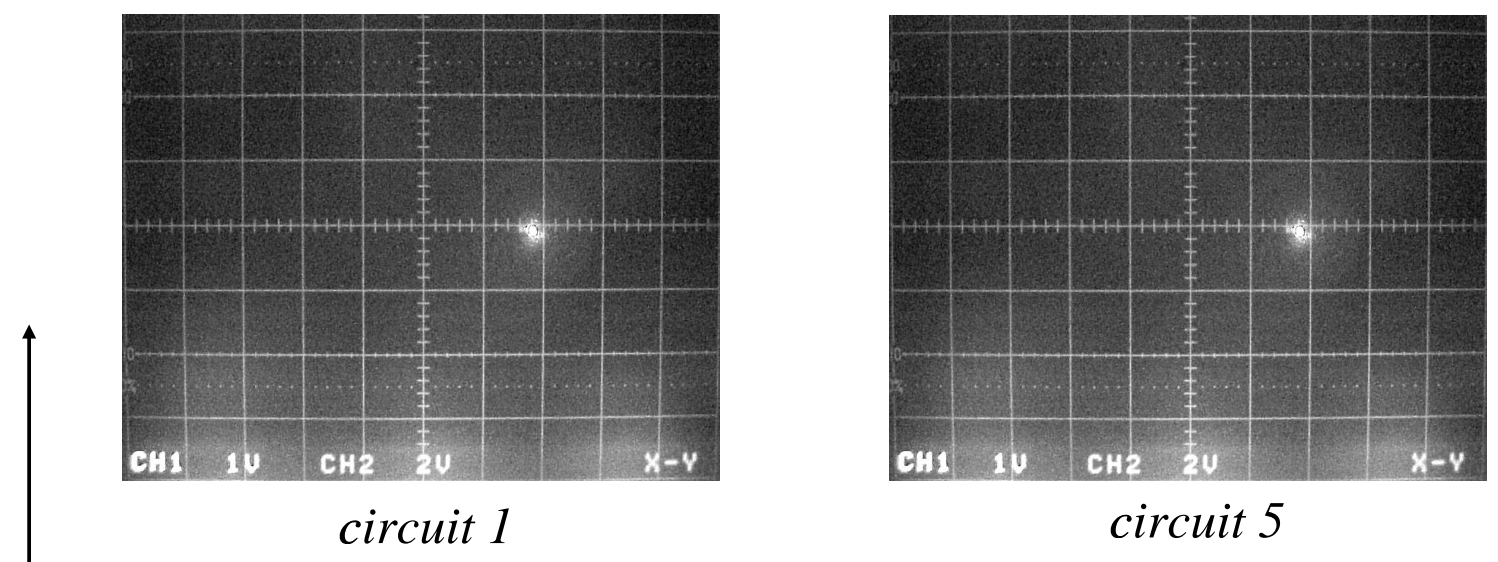

circuit 5

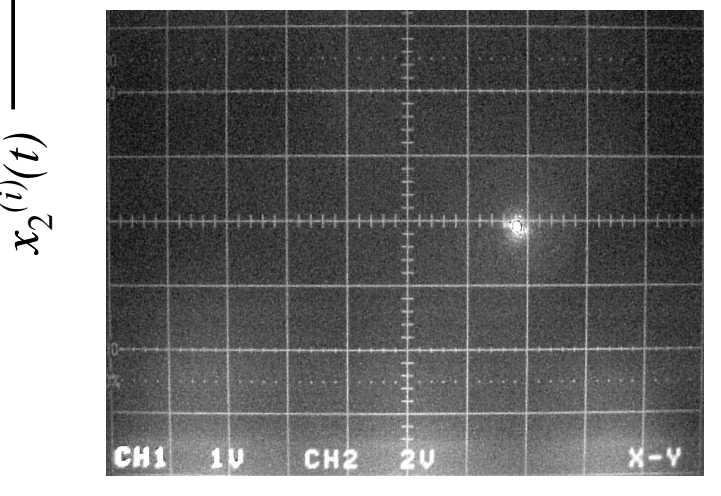

circuit 9



circuit 16

$x_{1}^{(i)}(t)$

(c)

Fig. 5. (Continued)

We then try to suppress the spatial instability. The gain resistor and the delay time of the controller are set to $R_{u}=2.0[\mathrm{k} \Omega]$ and $T=$ 3.96 [msec], respectively. In Fig. 4(a) the gain diagram of $\bar{G}(j \omega)$ is plotted by the broken line: we remark that the peak gain is less than 1 . According to the stability check, this result implies that we can make homogeneous solution (2) become TSS by the delayed feedback controller. Now, the controller in Fig. 3 is added to every circuit as shown in Fig. 1. Figure 4(c) shows behavior of the controlled circuits; the trajectories of all the circuits converge on the fixed point $\mathbf{P}$. From this result it may be stated that our controller succeeds in suppressing the spatial instability in the coupled circuits.

In addition, we try to stabilize chaotic behavior in TU regime by the same control method. For a parameter set $(R, r)=(1.8[\mathrm{k} \Omega], 8.0[\mathrm{k} \Omega])$, the stability check informs us that solution (2) is TU.
Figure 5(a) shows the circuit behavior for this parameter set. It can be seen that all the circuits oscillate chaotically. The controller parameters are set to $R_{u}=2.2[\mathrm{k} \Omega]$ and $T=3.5$ [msec]. Figure 5(b) is the gain diagram of $\bar{G}(j \omega)$; we notice that solution (2) will become TSS by the delayed feedback controller. The controlled circuit behavior shown in Fig. 5(c) suggests that our control method can also stabilize chaotic trajectories onto the fixed point $\mathbf{P}$.

\section{Conclusion}

In this letter, we proposed the decentralized delayed feedback controller to suppress the spatial instability in the one-way open coupled Chua's circuits. Electronic devices realized the coupled circuits and controllers. It has been shown experimentally that our controller can suppress the spatial instability and chaotic behavior in the coupled circuits. 


\section{Acknowledgments}

This research was partially supported by the Sasagawa Scientific Research Grant from Japan Science Society and by the Japan Society for the Promotion of Science (13750430, 2001).

\section{References}

Astakhov, V. V., Anishchenko, V. S. \& Shabunin, A. V. [1995] "Controlling spatiotemporal chaos in a chain of the coupled logistic maps," IEEE Trans. Circuits Syst.-I 42, 352-357.

Chen, G. \& Dong, X. [1998] From Chaos to Order (World Scientific, Singapore).

Fradkov, A. L. \& Pogromsky, A. Y. [1998] Introduction to Control of Oscillations and Chaos (World Scientific, Singapore).

Hu, G., Qu, Z. \& He, K. [1997] "Feedback control of chaos in spatiotemporal systems," Int. J. Bifurcation and Chaos 5, 901-936.

Imai, T., Konishi, K., Kokame, H. \& Hirata, K. [2002] "Experimental evidence for spatiotemporal stability and control of a one-way open CML," Int. J. Bifurcation and Chaos 12, 2937-2944.

Johnson, G. A., Löcher, M. \& Hunt, E. R. [1995] "Stabilized spatiotemporal wave in a convectively unstable open flow system: Coupled diode resonators," Phys. Rev. E51, 1625-1628.

Kaneko, K. [1985] "Spatial period-doubling in open flow," Phys. Lett. A111, 321-325.

Kaneko, K. [1993] Theory and Applications of Coupled Map Lattices (John Wiley).

Kapitaniak, T., Chua, L. O. \& Zhong, G. Q. [1994] "Experimental hyperchaos in coupled Chua's circuit," IEEE Trans. Circuits Syst.-I 41, 499-503.

Kennedy, M. P. [1994] "Robust OP amp realization of Chua's circuit," Frequenz 46, 66-80.

Konishi, K., Kokame, H. \& Hirata, K. [1998] "Decentralized delayed-feedback control of a coupled map model for open flow," Phys. Rev. E58, 3055-3059.

Konishi, K., Kokame, H. \& Hirata, K. [1999] "Stability of steady state in one-way open coupled map lattices," Phys. Lett. A263, 307-314.

Konishi, K., Kokame, H. \& Hirata, K. [2000a] "Delayedfeedback control of spatial bifurcations and chaos in open flow models," Phys. Rev. E62, 384-388.

Konishi, K., Kokame, H. \& Hirata, K. [2000b] "Spatiotemporal stability of one-way open coupled nonlinear systems," Phys. Rev. E62, 6383-6387.

Konishi, K., Kokame, H. \& Hirata, K. [2001] "Experimental evidence for spatiotemporal stability in oneway open coupled double-scroll circuits," IEEE Trans. Circuits Syst.-I 48, 1234-1237.

Konishi, K. [2002] "Spatiotemporal stability and control of one-way open coupled Lorenz systems," Phys. Rev. E65, 036203.

Pyragas, K. [1992] "Continuous control of chaos by selfcontrolling feedback," Phys. Lett. A170, 421-428.

Willeboordse, F. H. \& Kaneko, K. [1995] "Pattern dynamics of a coupled map lattice for open flow," Physica D86, 428-455.

Yamaguchi, A. [1997] "On the mechanism of spatial bifurcations in the open flow system," Int. J. Bifurcation and Chaos 7, 1529-1538.

\section{Appendix}

The $H_{\infty}$-norm of a transfer function is defined as follows.

Definition 1. Assume that a transfer function $G(s)$ is stable (i.e. $G(s)$ has all poles in left half plane on the complex plane). $H_{\infty}$-norm of $G(s)$ is defined as

$$
\|G(s)\|_{\infty}:=\sup _{\omega \in \Re}|G(j \omega)| .
$$

Roughly speaking, the $H_{\infty}$-norm is the maximum value of gain $|G(j \omega)|$ for angular velocity $\omega \in \mathbf{R}$, where $\omega=2 \pi f$. 